\title{
Molecular characterization of endophytes isolated from Saccharum spp based on esterase and ribosomal DNA (ITS1-5.8S-ITS2) analyses
}

\author{
A.C. Leme, M.R.R. Bevilaqua, S.A. Rhoden, C.A. Mangolin, \\ M.F.P.S. Machado and J.A. Pamphile \\ Departamento de Biotecnologia, Genética e Biologia Celular, \\ Universidade Estadual de Maringá, Maringá, PR, Brasil \\ Corresponding author: J.A. Pamphile \\ E-mail: japamphile@uem.br \\ Genet. Mol. Res. 12 (3): 4095-4105 (2013) \\ Received March 12, 2013 \\ Accepted July 29, 2013 \\ Published September 27, 2013 \\ DOI http://dx.doi.org/10.4238/2013.September.27.11
}

\begin{abstract}
This study used esterases and ribosomal DNA (rDNA) markers to determine endophytic variability in order to better understand endophyte-host interactions. Polyacrylamide gel electrophoresis and esterase isoenzymes (EST; EC 3.1.1.3), with $\alpha$-naphthyl acetate and $\beta$-naphthyl acetate as substrates, were used to assess relationships among endophytes. ITS1-5.8S-ITS2 sequencing data were used as rDNA markers. Thirty-two esterases were obtained from 37 isolates of Saccharum spp, which clustered into five endophyte groups. Esterase EST-06 was observed with the highest frequency, being present in 22 of the 37 isolates analyzed, followed by esterase EST-11, which was present in 20 isolates. The esterases EST-10 and EST-14 were present in 19 isolates and EST-09 was present in 18 isolates. The esterase EST-01 was unique to isolate 33 and can, therefore, be used as a marker for this isolate. None of the esterases identified were common to all isolates tested. Similarly, phylogenetic analysis, based on rDNA sequence data, classified the isolates into 5 genus groups: 1) Curvularia with a $100 \%$ bootstrap value (BP), 2) Alternaria with $100 \%$ BP, 3) Epicoccum with $60 \%$ BP, 4) Phoma with $89 \%$ BP, and 5)
\end{abstract}


Saccharicola with $100 \%$ BP. This polyphyletic analysis based on several markers, therefore, proved to be a valuable approach in determining the relationship between variation in endophytes and their associated host plants. Furthermore, both the esterase and rDNA analyses obtained similar results and were equally effective in resolving relationships.

Key words: Sugarcane; Endophytic fungi; Isoenzymes; Esterases; Molecular markers; rDNA

\section{INTRODUCTION}

Currently, Brazil is one of the largest sugar exporters globally, and serves as model in the use of ethanol as biofuel, attracting international interest with respect to technological development. The modern sugarcane cultivars used in commercial plantations are hybrid varieties with a high ploidy level, and new varieties are continuously being developed by agricultural institutes and livestock researchers to meet the increasing economic demand of this resource (Censo CTC, 2012).

Plants provide a complex micro-system with a wide variety of niches that offer suitable environments for the coexistence and interaction of diverse microorganisms, both in internal and external tissues. These species interactions may be affected by a number of internal and external factors affecting the plant habitat, including seasonal changes, the specific plant tissue, and the stage of development of the plant. Studies investigating various interactions between endophytic microorganisms and their plant hosts have only emerged in recent years. Microorganisms that are most commonly studied are those that live inside a host plant, do not produce external structures or cause damage to the plant, and can be readily propagated in culture medium (Azevedo and Araújo, 2007). All species of currently existing plants act as hosts for several endophytic species, although the details of most of these interactions remain unknown. Therefore, the probability of discovering new species of endophytes inhabiting plants is very high (Strobel and Daisy, 2003). Besides protection against abiotic stresses, these microorganisms also confer increased resistance to pathogen attack in their hosts (Arnold et al., 2003), and can be used as effective vectors for investigations of gene expression patterns in plants (Pamphile et al., 2004).

Most of the research conducted so far regarding beneficial plant-endophyte interactions has focused on endophytic fungi. Several species of fungi that are naturally found as endophytes are considered to be entomopathogenic, in which they act as parasites of insects and prevent disease symptoms. Such fungi have been observed in a diversity of plant species, such as banana, corn, cocoa and apple (Camatti-Sartori et al., 2005). Furthermore, because endophytic fungi have been shown to demonstrate high levels of phenotypic plasticity (Saikkonen at al., 1999), the role that they play in an ecosystem can vary substantially depending on both biotic and abiotic factors. The use of molecular markers has been useful in studies of endophytic variability, because these markers are capable of detecting polymorphisms in several species (Waldschimidt et al., 2002; Bernardi-Wenzel et al., 2010; Garcia et al., 2012; Orlandelli et al., 2012; Rhoden et al., 2012). Currently, the best tools available to detect endophyte variation effectively are ITS1-5. 8S-ITS2 sequences and isoenzymes. The most frequently used biochemical markers are esterases, a group of genetically distinct enzymes that are widely distributed among a diversity of taxa. Although they carry out a variety of functions in an organism, esterases share the property of catalyzing esters in hydrolysis, as well as pep- 
tides, amides and halides (Walker and Mackness, 1983).

Isoenzyme analysis has been successfully used to identify fungal species and in the differentiation of phytopathogenic fungi (Goodwin et al., 1995; Smith and Sivasithamparam, 2000; Láday et al., 2000). This technique provides a reliable method for the characterization and differentiation of endophytic fungi and their phytopathogens that is relatively fast, sensitive, and specific, without the need for further genetic or morphological analyses.

Considering the economic importance of endophytic fungi generally, and their relevance for the sugarcane industry of Brazil in particular, information regarding relationships between these organisms and their host plants is extremely valuable. This study aimed to establish esterase isoenzyme standards from a sample of the endophytic fungi community isolated from the leaves of sugarcane plants. Furthermore, these markers were used for molecular analysis to determine the evolutionary relationships among the isolates, and the isoenzyme results were compared with a phylogenetic analysis based on ribosomal DNA (rDNA) markers.

\section{MATERIAL AND METHODS}

\section{Host plant}

The endophytic fungi used in this study were isolated from sugarcane, Saccharum spp, leaves (varieties RB 855536 and SP 891115). Leaves that exhibited no signs of branding, injury, or stains were preferentially selected.

\section{Obtaining endophytic fungi from Saccharum spp}

Sugarcane leaves were collected at the São Luiz Factory, Ourinhos, São Paulo. We harvested leaves of the RB 855536 and SP 891115 varieties, which are used by this agroindustry as raw materials for the production of sugar and alcohol. After collection, the leaves were packaged in plastic bags and transported in ice boxes to the laboratory, where they were cleaned with water and transferred to new plastic bags.

\section{Isolation of endophytic fungi}

Superficial disinfection of the collected leaves was carried out using sodium hypochlorite (NaClO), according to protocols described in Bernardi-Wenzel et al. (2010), with slight modifications: leaves were disinfected in $5 \% \mathrm{NaClO}$ for 5 min.

After the superficial disinfection, the epiphytic community was removed by first aseptically cutting the leaves into small fragments of approximately $4 \times 2 \mathrm{~mm}$. Next, four leaf fragments were placed onto separate Petri dishes containing half-strength potato dextrose agar (PDA) medium and $50 \mu \mathrm{g} / \mathrm{mL}$ tetracycline to prevent bacterial growth. The plates were incubated at $28^{\circ} \mathrm{C}$ for five days. The fungi grown were then transferred to new PDA plates and purified.

\section{Grouping, characterization and identification of endophytic fungi based on morphology}

The isolated fungi were transferred to solid PDA media using the prick technique and incubated at $28^{\circ} \mathrm{C}$. After seven days, they were grouped according to colony appearance, 
color and mycelium type. The micro-culture plate technique was used for the identification of microscopic structures using a light microscope, according to methods described in Kern and Blevins (1999). The data from macroscopic and microscopic (cytological) analyses were compared with identification keys and results of the molecular analysis (rDNA sequencing).

\section{DNA extraction}

DNA extraction was carried out using the Ultra-Clean Microbial DNA isolation Kit (MO BIO Laboratories Inc.) according to manufacturer instructions.

DNA concentration was estimated by electrophoresis on $1.0 \%$ agarose gel. High DNA Mass Ladder (Invitrogen) was used as the molecular weight standard. After electrophoresis, the gel was photographed and bands were analyzed.

\section{DNA amplification by polymerase chain reaction (PCR)}

Ribosomal DNA amplification of the ITS1-5.8S-ITS2 region was performed, using the primers ITS1 and ITS4, as described in White et al. (1990).

\section{Purification of the amplification products}

The ITS1-5.8S-ITS2 amplified regions of rDNA from the isolates were purified using the GFX PCR DNA and Gel Band Purification Kit (Amersham Biosciences), according to the manufacturer instructions. After purification, the DNA of the endophytic isolates was quantified on $1 \%$ agarose gel, photographed and bands analyzed.

\section{Sequencing of the ITS regions of rDNA}

DNA samples corresponding to the ITS1-5. 8S-ITS2 rDNA region were sequenced by PCR to a final volume of $8 \mu \mathrm{L}(1 \mu \mathrm{L}$ ITS1 primer, $2 \mu \mathrm{L}$ previously purified DNA, and 3 $\mu \mathrm{L}$ Milli-Q water) in a $2 \mu \mathrm{L}$ DYEnamic ${ }^{\mathrm{TM}}$ ET dye Terminator Cycle Kit $\left(\mathrm{MegaBACE}^{\mathrm{TM}}\right)(\mathrm{GE}$ Healthcare). The reaction mixture was then placed in a thermal cycler (MJ Research, Inc.TTC100), programmed to carry out 35 cycles after an initial denaturation at $95^{\circ} \mathrm{C}$ for $2 \mathrm{~min}$. Each cycle consisted of three stages: denaturation at $95^{\circ} \mathrm{C}$ for $1 \mathrm{~min}$, annealing at $55^{\circ} \mathrm{C}$ for $1 \mathrm{~min}$ and extension at $60^{\circ} \mathrm{C}$ for $1 \mathrm{~min}$. A final cycle was run at $60^{\circ} \mathrm{C}$ for $5 \mathrm{~min}$. The sequencing was performed in a MegaBACE ${ }^{\mathrm{TM}} 1000$ sequencer (Amersham Biosciences). The injection and electrophoresis conditions were $1 \mathrm{Kv} / 90 \mathrm{~s}$ and $7 \mathrm{Kv} / 240 \mathrm{~min}$, respectively.

After sequencing, the samples were analyzed and edited. To identify the isolates, obtained nucleotide sequences were compared to those deposited in the National Center for Biotechnology Information (NCBI) database. The BLAST program (http://www.ncbi.nlm.nih. gov/blast/) was used to identify species. The nucleotide sequences obtained in this study have been submitted to GenBank and were assigned accession numbers JX416911 to JX416919.

\section{Estimation of genetic distance of the isolates}

The sequences determined were aligned using the program MEGA (version 5.0) 
(Tamura et al., 2011) and were classified by the neighbor-joining (NJ) method (Saitou and Nei, 1987), using a p-distance matrix for nucleotides with the pairwise gap deletion option and 10,000 bootstrap repetitions.

\section{Isoenzymes (esterases) analysis}

For the extraction of esterases, 37 selected isolates of endophytic fungi were cultivated in Petri dishes on PDA culture media for 7 days at $28^{\circ} \mathrm{C}$. Approximately $5 \mathrm{~mm}$ mycelial fragments of each of the 37 isolates were removed, rinsed with distilled water and placed in a microcentrifuge tube with $20 \mu \mathrm{L}$ cold extraction solution, containing $1.0 \mathrm{M}$ phosphate buffer, $\mathrm{pH} 7.0,5 \%$ PVP-40, $1.0 \mathrm{mM}$ ethylenediaminetetraacetic acid (EDTA), $0.5 \% \beta$-mercaptoethanol, and $10 \%$ glycerol. The entire crushing procedure was performed in an ice bath. After homogenization, the samples were centrifuged in a Hettich MIKRO 220R centrifuge for $30 \mathrm{~min}$ at $14,000 \mathrm{rpm}$ and $4^{\circ} \mathrm{C}$.

The supernatants were used as samples for polyacrylamide gel electrophoresis. The $12 \%$ polyacrylamide gel was prepared with $0.375 \mathrm{M}$ Tris- $\mathrm{HCl}, \mathrm{pH}$ 8.8. Electrophoresis was performed for $5 \mathrm{~h}$ at $4^{\circ} \mathrm{C}$ with a constant voltage of $200 \mathrm{~V}$ and $0.1 \mathrm{M}$ Tris/glycine buffer, $\mathrm{pH} 8.3$, was used in the chamber. The polyacrylamide gels were prepared following protocols described in Ceron (1988).

To identify the $\alpha$ - and $\beta$-esterase activities, the gel was incubated with substrate-specific dyes for each enzyme. To detect the $\alpha$ - and $\beta$-esterases, the gel was incubated in a 60 $\mathrm{mL}$ solution containing $0.1 \mathrm{M}$ sodium phosphate, $\mathrm{pH} 6.2,60 \mathrm{mg} \alpha$-naphthyl acetate, $60 \mathrm{mg}$ $\beta$-naphthyl acetate, and $60 \mathrm{mg}$ Fast Blue RR Salt dye dissolved in $1 \mathrm{~mL}$ N-propanol. After staining, the gels were fixed at room temperature for $1-24 \mathrm{~h}$ in $7.5 \%$ acetic acid solution and $10 \%$ glycerol. After fixation, the gel was dried with $5 \%$ gelatin and placed between two stretched sheets of cellophane paper for 24-48 $\mathrm{h}$.

\section{RESULTS AND DISCUSSION}

\section{Isolation of endophytic fungi from Saccharum spp}

A total of 400 leaf fragments were collected from Saccharum spp, 79.25\% of which successfully colonized. A total of 425 endophytic fungi were apparent on PDA, and 97 of these fragments were randomly selected to be isolated for further analysis. These isolates were grouped into seven morph groups, according to variations in colony color, development, and growth on PDA. The lack of growth in the negative control assured efficiency of the disinfection process. Thirty-seven endophytes, including representatives from each morph group, were chosen randomly and purified for use in experiments involving esterase analysis.

\section{Molecular identification of isolates based on rDNA sequencing}

Identification of the genera and species of 9 isolates belonging to 7 different morph groups of endophytic fungi was achieved, based on a BLAST search in the NCBI database with the obtained ITS1-5. 8S-ITS2 sequences. After sequencing the fungi, five endophytic fungus genera were identified: Epicoccum, Dothideales, Curvularia, Saccharicola and Alternaria (Table 1). Five out of the 7 morph groups were represented in the Saccharum spp cultivars colonized by endophytes, based on the identification and determination of differential 
expression of esterases: morph group 1 (Curvularia sp and Saccharicola sp); morph group 2 (Dothideales sp); morph group 3 (Saccharicola sp and Alternaria alternata); morph group 5 (Epicoccum sp); morph group 7 (Epicoccum nigrum). The micro-culture technique, using a subset of the samples, confirmed the results obtained by ITS1-5. 8S-ITS2 sequencing.

\begin{tabular}{|c|c|c|c|}
\hline Endophyte isolated & Genera/species & NCBI accession No. & Identity (\%) \\
\hline Isol. 12 & Epicoccum nigrum & FJ605251 & 98 \\
\hline Isol. 15 & Epicoccum sp & HQ914874 & 96 \\
\hline Isol. 18 & Dothideales $\mathrm{sp}$ & HQ608003 & 96 \\
\hline Isol. 33 & Saccharicola sp & GQ370379 & 96 \\
\hline Isol. 34 & Curvularia sp & HQ909079.1 & 99 \\
\hline Isol. 43 & Saccharicola sp & GU973718.1 & 95 \\
\hline Isol. 48 & Alternaria alternata & EF104192.1 & 98 \\
\hline Isol. 53 & Saccharicola sp & GU973718 & 94 \\
\hline Isol. 57 & Saccharicola sp & GQ370379.1 & 99 \\
\hline
\end{tabular}

\section{Phylogenetic analysis of Saccharum spp endophytes based on rDNA sequence data}

Phylogenetic analysis classified the rDNA sequences into 5 separate genus groups (Figure 1): 1) Curvularia, with a 100\% bootstrap (BP) value, 2) Alternaria, 100\% BP, 3) Epicoccum, 60\% BP, 4) Phoma, 89\% BP and 5) Saccharicola, 100\% BP.

Overall, classifications based on the phylogenetic analysis confirmed the results obtained by BLAST analysis of the sequence data. The endophyte isolate 34 (99\% identity to Curvularia sp HQ909079.1 in the BLAST analysis) was grouped into group 1, Curvularia (75\% BP), and was associated with a subgroup of Curvularia sp (HM775181) and C. geniculate (JN006774). Similarly, the endophyte isolate 48 showed 98\% identity with Alternaria alternata EF104192.1 in the BLAST analysis, and was grouped with the fungus species $A$. Alternata $(100 \% \mathrm{BP})$ in the phylogenetic analysis.

The isolates 12 (98\% identity with E. nigrum FJ605251 in the BLAST analysis) and 15 (96\% identity with Epicocum sp in the BLAST analysis) were grouped with the fungal species Epicoccus sp (HQ919872) with 65\% BP, resulting in confirmation of their genus identifications. The endophyte isolate 18 (96\% identity with Dothideales sp HQ608003 in the BLAST analysis) was grouped with the genus Phoma sp with $89 \%$ BP, which confirmed that these isolates were most likely members of the genus Phoma.

The endophyte isolates 53 (94\% identity with Saccharicola sp GU973718 in the BLAST analysis), 33 (96\% identity with Saccharicola sp GQ370379 in the BLAST analysis), 57 (99\% identity with Saccharicola GQ370379.1 in the BLAST analysis) and 43 (95\% identity with Saccharicola sp GU973718.1 in the BLAST analysis) were confirmed as belonging to the genus Saccharicola with 100\% BP.

\section{Evaluation of genetic diversity of endophyte isolates based on isoenzymes}

Esterases are a group of hydrolase enzymes that split esters. They are present in all organisms, and also commonly used as non-specific markers in genetics studies, since they are easy to detect and are highly polymorphic (Davis, 1964). Substrate specificity has been observed in 


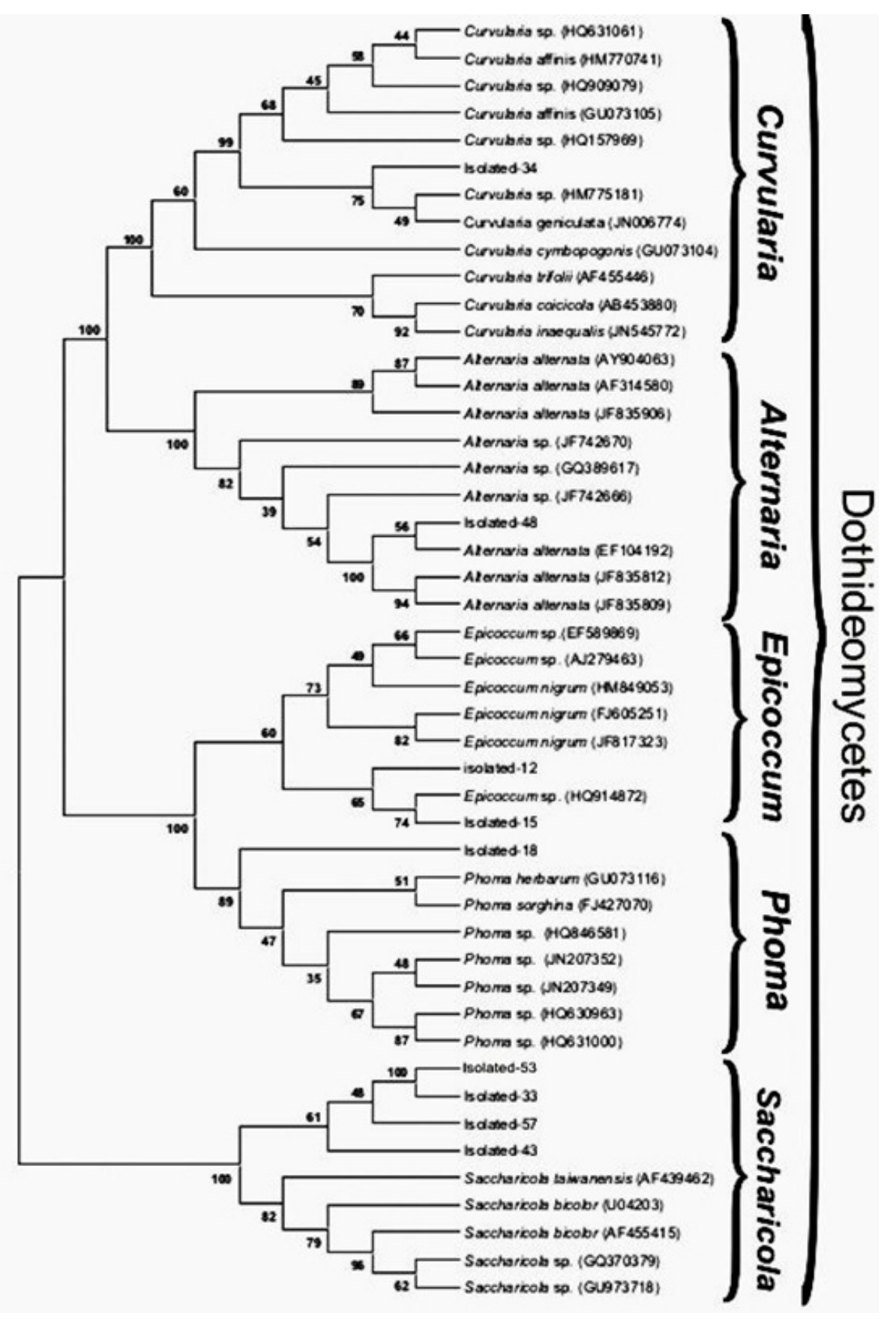

Figure 1. Phylogenetic tree constructed with sequences of the ITS1-5.8S-ITS2 of rDNA region of endophytic fungi from Saccharum spp and sequences from GenBank (indicated by database code), using the neighbor-joining method and using p-distance for nucleotides, with the pairwise gap deletion option. The numbers above and underneath each knot indicate the frequency (in percentage) of each branch in bootstrap analyses of 10,000 replicates. 
most esterases, indicating that they may have extensive biological functions. Esterases have been extensively studied in insects, where they have been shown to be involved in various physiological processes, including as regulators of juvenile hormone levels (Kort and Granger, 1981), in reproduction (Mane et al., 1983), nervous system function, and in the development of resistance to insecticides (Fournier et al., 1993). In plants, the differential expression of isoesterases have been used as a bacterial infection marker following pathogenesis (Pereira et al., 2001). However, esterase studies in vertebrates and microbes are sparse (Oakeshott et al., 1993).

From the 37 isolates of endophytic fungi obtained from Saccharum spp, 32 esterase loci were obtained, which could be classified into 6 groups. Esterase EST-06 was found with the highest frequency, appearing in 22 of the 37 isolates analyzed, followed by esterase EST11, which was present in 20 isolates. The esterases EST-10 and EST-14 were present in 19 isolates, and EST-09 was present in 18 isolates. Esterase EST-01 was found uniquely in isolate 33 and can therefore serve as a useful marker of this isolate.

None of the esterases identified were common to all strains tested (Figure 2).

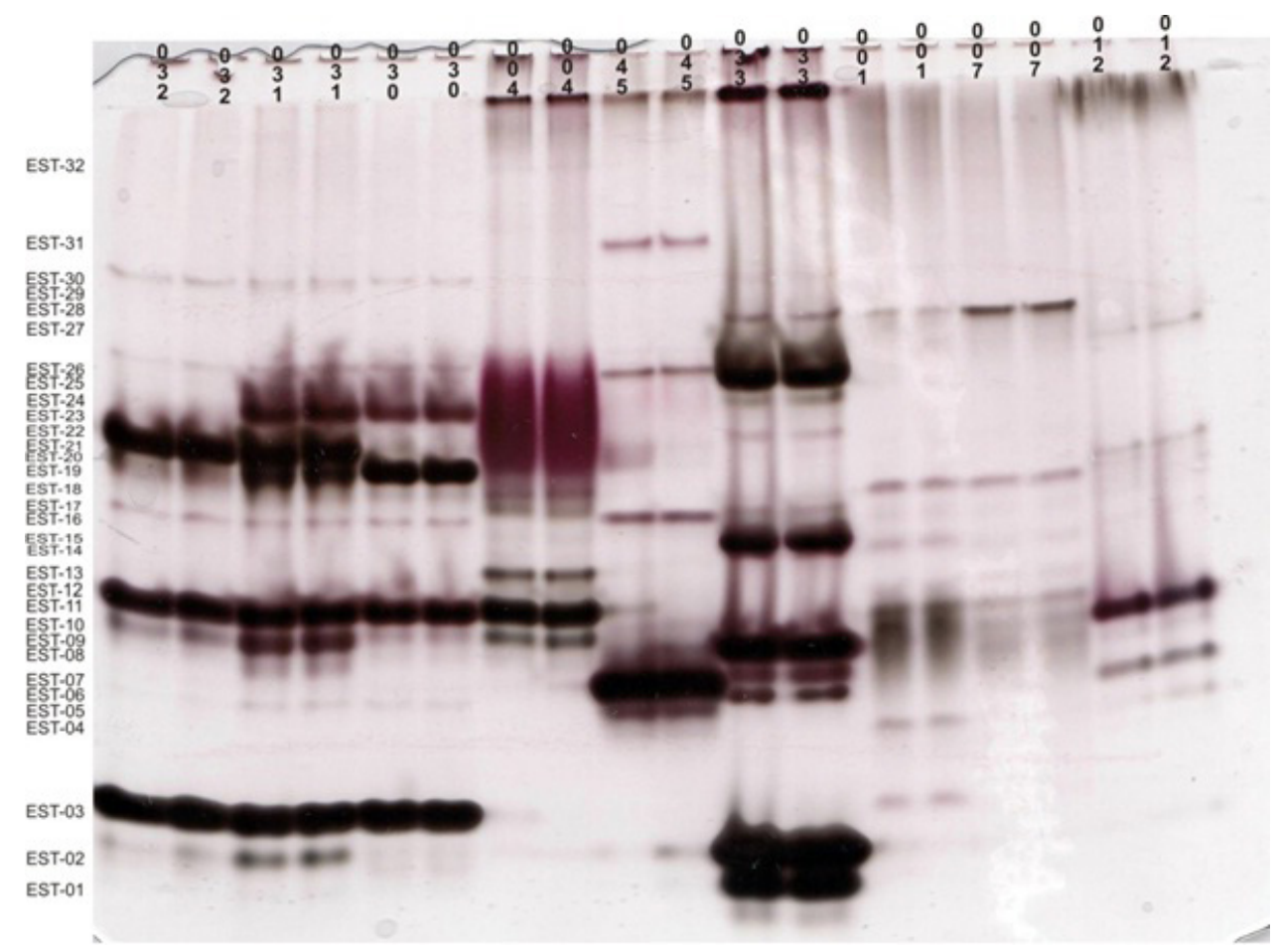

Figure 2. Polymorphism of $\alpha$ - and $\beta$-esterases produced for the 32 esterases detected in 37 different endophytic fungi isolated from leaves of sugarcane. Esterases evaluated 9 different isolates.

After examining gels for the presence or absence of esterases, a binary data matrix was built and used to calculate genetic distances among the isolates. The constructed dendrogram is shown in Figure 3. The isolates were separated into 6 different groups. Group one (G.1) consisted of endophytic isolates 01, 02, 03, 04, 07, 09, 10, 11, 12 (Epicoccum nigrum), 13, 15 (Epicoccum sp), 19, 21, 45, and 53 (Saccharicola sp). Within this group, two subgroups were observed that 
showed high levels of genetic similarity between isolates 11 and $13(0.9688)$ and between isolates 11 and 15 (Epicoccum sp) (0.9375). Group 2 (G.2) consisted of isolates 24, 28, 34 (Curvularia sp), 35, 36, 41, 42, 43 (Sacchariola sp), 48 (Alternaria alternata), 51, and 57 (Sacchariola sp), with subgroups composed of isolates 36 and 57 (1.0000) and isolate 35 (0.9688). Group 3 (G.3) included the isolates 17, 20,22, 25, and 39. In this group, the highest genetic similarity was observed between isolates 17 and 22 (0.9688) and between isolates 25 and $39(0.9062)$.

The 4th group (G.4) obtained by the isoenzyme analysis was formed by the isolates 30,31 , and 32. Group 5 was formed by isolates 18 and 27. The isolate 33 (Sacchariola sp) was not associated with any other group formed by the isoenzymes. The lowest genetic similarity (0.3438) amongst the 37 isolates evaluated was observed between the isolates 27 (morphogroup 02 ) and 48 (Alternaria alternata; morphogroup 03). The highest genetic similarity (1.0000) was observed between isolates 36 and 57 (Sacchariola sp), both belonging to morphogroup 01 .

These results showed that the isolates form six distinct groups. Similarly, Banke et al. (1996) used isoenzymes for a taxonomic analysis of 84 isolates of Penicillium chrysogenum and related species. Four groups were defined, which were suggested to represent four different species based on their genetic distances. Using 26 enzyme systems, Láday et al. (2000) investigated polymorphism among 34 isolates of Fusarium graminearum. The results obtained classified the enzymes into two different groups based on their respective geographic origins. The authors suggested that isolates within a given group descended from a common ancestral population and that isoenzymes from the two groups had different ancestors.

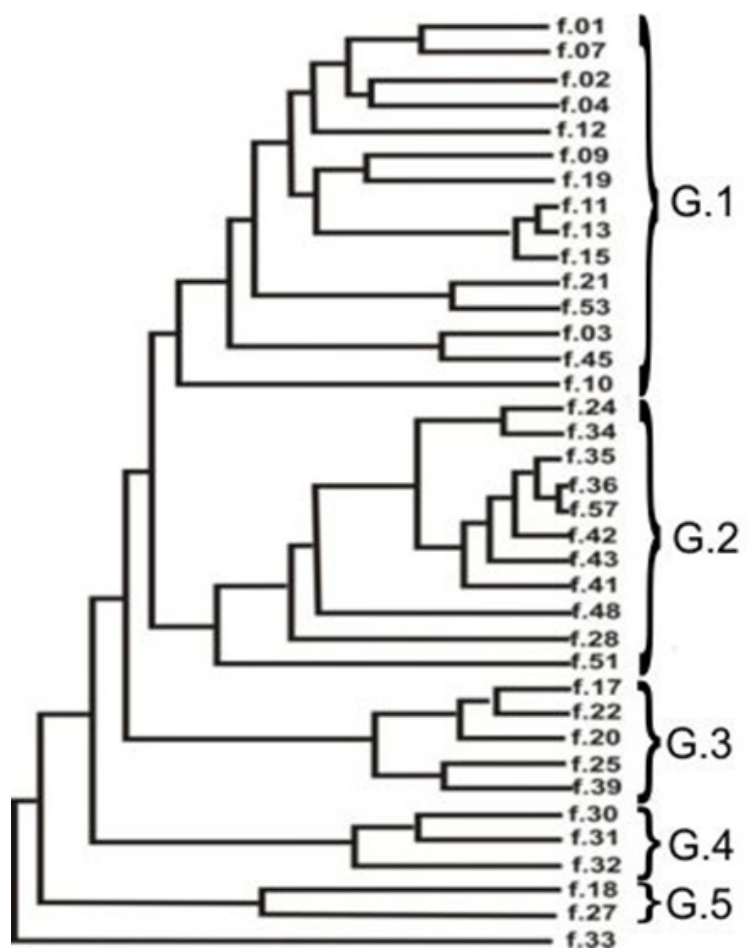

Figure 3. Dendrogram representing the relationship between the endophytic fungi isolated from leaves of sugarcane based on measures of genetic distance obtained by UPGMA. 
In summary, endophytes of Saccharum spp were classified into 7 morphogroups based on morphological analysis, 6 genetic groups based on esterase analysis, and 5 genetic groups based on phylogenetic analysis using ITS1-5.8S-ITS2 markers. These results demonstrate the value of conducting polyphyletic analyses based on several markers in order to understand variability in endophyte-plant host associations. Furthermore, esterase and rDNA analyses revealed similar results and are therefore both valuable tools for these types of analyses.

\section{ACKNOWLEDGMENTS}

To CAPES (Coordenação de Aperfeiçoamento de Pessoal de Ensino Superior) for master's scholarship and PhD's scholarship.

\section{REFERENCES}

Arnold AE, Mejía LC, Kyllo D, Rojas EI, et al. (2003). Fungal endophytes limit pathogen damage in a tropical tree. PNAS 26: $15649-15654$.

Azevedo JL and Araújo WL (2007). Diversity and Applications of Endophytic Fungi Isolated from Tropical Plants. In: Fungi: Multifaceted Microbes (Ganguli BN and Desmuckh SK, eds.). Boca Raton, Anamaya Publishers and CRC Press, v. 01, 189-207.

Banke S, Frisvad JC and Rosendahl S (1996). Taxonomy of Penicillium chrysogenum and related xerophilic species, based on isoenzymes analysis. Mycol. Res. 101: 617-624.

Bernardi-Wenzel J, Garcia A, Filho CJ, Prioli AJ, et al. (2010). Evaluation of foliar fungal endophyte diversity and colonization of medicinal plant Luehea divaricata (Martius et Zuccarini). Biol. Res. 43: 375-384.

Camatti-Sartori V, da Silva-Ribeiro RT, Valdebenito-Sanhueza RM, Pagnocca FC, et al. (2005). Endophytic yeasts and filamentous fungi associated with southern Brazilian apple (Malus domestica) orchards subjected to conventional, integrated or organic cultivation. J. Basic Microbiol. 45: 397-402.

Censo - CTC (2012). Censo Varietal e de Produtividade, Agosto 2012. Available at [http://www.CTCanavieira.com.br]. Accessed September 9, 2012.

Ceron CR (1988). Padrão de Esterases no Desenvolvimento de Drosophila mulleri, D. arizonensis e seus Hibridos. Doctoral thesis, Instituto de Biociências, Universidade de São Paulo, USP, São Paulo.

Davis BJ (1964). Disc electrophoresis. ii. method and application to human serum proteins. Ann. N. Y. Acad. Sci. 121: 404-427.

Fournier D, Mutero A, Pralavorio M and Bride JM (1993). Drosophila acetylcholinesterase: mechanisms of resistance to organophosphates. Chem. Biol. Interact. 87: 233-238.

Garcia A, Rhoden SA, Rubin-Filho CJ, Nakamura CV, et al. (2012). Diversity of foliar endophytic fungi from the medicinal plant Sapindus saponaria L. and their localization by scanning electron microscopy. Biol. Res. 45: 139-148.

Goodwin SB, Schneider RE and Fry WE (1995). Use of cellulose-acetate electrophoresis for rapid identification of allozyme genotypes of Phytophthora infestans. Plant Dis. 79: 1181-1185.

Kern ME and Blevins KS (1999). Micologia Médica. $2^{\circ}$ Edição. Editora Premier, São Paulo, 256.

Kort CA and Granger NA (1981). Regulation of the juvenile hormone titer. Ann. Rev. Entomol. 26: 1-28.

Láday M, Bagi F, Mesterházy A and Szécsi Á (2000). Isoenzymes evidence for two groups of Fusarium graminearum. Mycol. Res. 104: 788-793.

Mane SD, Tompkins L and Richmond RC (1983). Male esterase 6 catalyzes the synthesis of a sex pheromone in Drosophila melanogaster females. Science 222: 419-421.

Oakeshott JG, van Papenrecht EA, Boyce TM, Healy MJ, et al. (1993). Evolutionary genetics of Drosophila esterases. Genetica 90: 239-268.

Orlandelli RC, Alberto RN, Rubin Filho CJ and Pamphile JA (2012). Diversity of endophytic fungal community associated with Piper hispidum (Piperaceae) leaves. Genet. Mol. Res. 11: 1575-1585.

Pamphile JA, Rocha CLMSC and Azevedo JL (2004). Co-transformation of a tropical maize endophytic isolate of Fusarium verticillioides (synonym F. moniliforme) with gusA and nia genes. Genet. Mol. Biol. 27: 253-258.

Pereira AJ, Lapenta AS, Vidigal-Filho PS and Machado MF (2001). Differential esterase expression in leaves of Manihot esculenta Crantz infected with Xanthomonas axonopodis pv. manihotis. Biochem. Genet. 39: 289-296. 
Rhoden SA, Garcia A, Rubin Filho CJ, Azevedo JL, et al. (2012). Phylogenetic diversity of endophytic leaf fungus isolates from the medicinal tree Trichilia elegans (Meliaceae). Genet. Mol. Res. 11: 2513-2522.

Saikkonen K, Helander M, Faeth SH, Schulthess F, et al. (1999). Endophyte-grass-herbivore interactions: the case of Neotyphodium endophytes in Arizona fescue populations. Oecologia 121: 411-420.

Saitou N and Nei M (1987). The neighbor-joining method: a new method for reconstructing phylogenetic trees. Mol. Biol. Evol. 4: 406-425.

Smith BJ and Sivasithamparam K (2000). Isozymes of Ganoderma species from Australia. Mycol. Res. 104: 952-961.

Strobel G and Daisy B (2003). Bioprospecting for microbial endophytes and their natural products. Microbiol Mol. Biol. Rev. 67: 491-502.

Tamura K, Peterson D, Peterson N, Stecher G, et al. (2011). MEGA5: molecular evolutionary genetics analysis using maximum likelihood, evolutionary distance, and maximum parsimony methods. Mol. Biol. Evol. 28: 2731-2739.

Waldschmidt AM, De Marco PJR, Barros EG and Campos LAO (2002). Genetic analysis of Melipona quadrifasciata Lep. (Hymenoptera: Apidae, Meliponinae) with RAPD markers. Braz. J. Biol. 62: 923-928.

Walker CH and Mackness MI (1983). Esterases: problems of identification and classification. Biochem. Pharmacol. 32: 3265-3269.

White TJ, Bruns TD, Lee S and Taylor JW (1990). Amplification and Direct Sequencing of Fungal Ribosomal RNA Genes for Phylogenetics. In: PCR Protocols: A Guide to Methods and Applications (Innis MA, Gelfand DH, Sninsky JJ and White TJ, eds.). Academic Press, San Diego, 315-322. 